\title{
Intestinal dysbacteriosis leads to kidney stone disease
}

\author{
ENYANG ZHAO, WENFU ZHANG, BO GENG, BOSEN YOU, WANHUI WANG and XUEDONG LI
}

Department of Urology, The Second Affiliated Hospital of Harbin Medical University, Harbin, Heilongjiang 150086, P.R. China

Received June 6, 2020; Accepted November 27, 2020

DOI: $10.3892 / \mathrm{mmr} .2020 .11819$

\begin{abstract}
The formation and physicochemical properties of kidney stones (KSs) are closely associated with diet. In view of the differences in ethnicity and dietary composition between Chinese and Western populations, the present study aimed to investigate the association between intestinal dysbacteriosis and KSs in China. The current study examined the differences in intestinal microbes between the KS disease (KSD) and the healthy control (HLT) groups, and statistically significant differences based on 16s rRNA gene amplicons were identified using a Student's t-test or one-way ANOVA. In addition, the calcium oxalate KS (COKS), uric acid KS (UAKS) and carbonate apatite $\mathrm{KS}(\mathrm{CCKS})$ groups were compared with a non-parametric statistical test. Determination of bacterial abundance was performed via the analysis of 16s rRNA marker gene sequences using next-generation sequencing. Firmicutes (F) and Bacteroides (B) levels were significantly higher in the KSD group compared with the HLT group $(\mathrm{B} / \mathrm{F}=0.67$ vs. $0.08 ; \mathrm{P}<0.001)$, as were the overall levels of $\mathrm{B}$ (6.19-fold higher compared with the HLT group; 22.2 vs. $3.6 \%$; $\mathrm{P}<0.001)$. The Prevotella-9 abundance levels in the KSD group were 4.65 -fold higher compared with those in the HLT group (8.8 vs. $1.9 \%$; $\mathrm{P}<0.001$ ). The levels of Blautia and Lachnoclostridium were significantly decreased in the KSD group (13.3 vs. $6.0 \%$; and 5.0 vs. $7.9 \%$; both $\mathrm{P}<0.05$ ). Moreover, Prevotella-9 levels were higher in non-calciferous KSs (UAKS) compared with calciferous KSs (COKS and $\mathrm{CCKS}$ ). Therefore, the findings of the present study indicated a key association between specific KS components and intestinal flora, providing a theoretical basis for new treatment methods for KSs. Moreover, differences and interactions between these bacteria could initially predict specific types of urolithiasis.
\end{abstract}

\section{Introduction}

Kidney stone (KS) disease (KSD) is one of the most common urological diseases. In Western countries, $5-10 \%$ of the

Correspondence to: Dr Xuedong Li, Department of Urology, The Second Affiliated Hospital of Harbin Medical University, 246 Xuefu Road, Harbin, Heilongjiang 150086, P.R. China

E-mail: 13604803553@163.com

Key words: intestinal dysbacteriosis, kidney stone disease population experience $\geq 1$ urinary calculus episode during their lifetime $(1,2)$. KSD is the result of metabolic disorders that occur for multiple reasons, but its exact pathogenesis remains elusive. Genetic variations, dietary habits and geographic distributions all contribute to the formation of KSs (3), with diet being an important factor. Different global regions and dietary cultures result in complex and diverse stone compositions, including calcium oxalate, calcium phosphate, calcium carbonate, cysteine and uric acid stones $(2,3)$. The incidence of KSD may be decreased by changing to a low-protein and low-salt diet, and by increasing the intake of fruit, whole grain cereal fiber and magnesium (4-6).

Eating habits and types of food can directly affect intestinal micro-organisms. Numerous studies (2,3,5,7-15) have reported that intestinal microbes can participate in the synthesis and metabolic breakdown of molecules involved in physiological and pathophysiological processes, including short-chain fatty acids, amino acids, vitamins, neurotransmitters and hormones, amongst others (7). Changes in metabolites or the induction of the immune response due to the disruption caused by intestinal dysbacteriosis may contribute to the regulation of various diseases. For example, short-chain fatty acids secreted by intestinal bacteria affect the metabolism of foreign substances, branched-chain amino acid transport, oxidative stress and the mucin degradation mechanism that regulates obesity and diabetes (7-13). Overconsumption of choline or carnitine generates trimethylamine (TMA) during intestinal metabolism, which subsequently produces TMA N-oxide (TMAO). The presence of TMAO promotes platelet activation and increases the incidence of atherosclerosis (14-16). Thus, blocking the intestinal TMA metabolic enzyme may significantly inhibit atherosclerotic plaque formation (15). These findings suggest that intestinal microbial disorders may be the cause of disease onset and are not only associated with disease symptoms.

Stern et al (2) used the 16s RNA method to determine the presence of intestinal microbes in 23 cases of KSs, and the levels of Bacteroides and Prevotella in the experimental group were found to be significantly increased. In addition, Suryavanshi et al (17) reported that the level of Oxalobacter in the feces was lower in the KSD group compared with that in the healthy control group (HLT), and this lower abundance was observed among patients with KSD with oxalate metabolism disorder and calcium oxalate components. These aforementioned results suggest that calcium oxalate stones in Western populations may be caused by i) a close correlation between intestinal dysbacteriosis and the incidence of 
KSD, and ii) low levels of Oxalobacter in the intestinal tract. Since diets differ between Chinese and Western populations, KSs exhibit marked diversity and no significant distribution characteristics, and therefore, it remains unknown whether different intestinal bacterial disorders may lead to the formation of different types of KSs. Thus, the aim of the present study was to analyze the diversity of intestinal flora in KSD and HLT subjects in northeastern China, and further analyze the variability of intestinal flora and gene abundance among patients with calcium oxalate KS (COKS), uric acid KS (UAKS) and carbonated apatite KS (CCKS), in order to investigate the association between the different types of KSs and intestinal dysbacteriosis. The findings of the present study may enable the design of KS preventive treatments by control of the intestinal flora via diet, precise medical treatment of KS and relatively cost-effective simple intestinal flora sequencing analysis paired with diet intervention.

\section{Materials and methods}

Ethics statement. The protocol of the present study was approved by the Ethics Committee of the Second Affiliated Hospital of Harbin Medical University (approval no. 2015-yan-221) and complied with the World Medical Association Declaration of Helsinki regarding ethical conduct of research involving human subjects. The study was performed in accordance with the approved local guidelines and regulations, and written informed consent was obtained from all the participants.

Patients. The study involved 91 individuals with KSD (age, 26-80 years; 48 male patients and 43 female patients), including 42 with COKS, 12 with UAKS and 18 with CCKS, and 75 HLT (age, 21-85 years; 44 male patients and 31 female patients). Stone samples were only collected from the patients who were treated with percutaneous nephrolithotomy. It was not possible to collect stone samples from the remainder of the patients who were treated with holmium laser lithotripsy. The participants provided blood samples $(5 \mathrm{ml})$, urine samples (10 ml) and stool samples (using sterile cryopreserved tubes) after $6 \mathrm{~h}$ of fasting. The fresh samples were stored in a freezer at $-80^{\circ} \mathrm{C}$ until analysis. All samples were collected from individuals who had not undergone gastrointestinal surgery and before the use of antibiotics. Patients with KSD included both those with newly developed and those with recurrent KSs. All stone composition types were registered. Classification of stones was based on the components with the largest proportions (18). HLT participants had not used medications over the past year, and those who had undergone gastrointestinal surgery or received antibiotics within the past 2 weeks were excluded. The samples were collected by two surgical methods, percutaneous nephrolithotomy and holmium laser lithotripsy. Stone specimens of the patients were successfully collected and stone analysis was conducted via percutaneous nephrolithotomy. However, the stone was divided into 1-2 mm size after holmium laser lithotripsy and failed to be successfully collected, which is the reason for incomplete samples.

Gut microbiome analysis. Fecal samples $(100 \mu \mathrm{l})$ were collected in Specimen Transport Medium (Qiagen, Inc.) and microbiome DNA was extracted using two methods, with a bead-beating based PowerSoil DNA isolation kit (MO BIO Laboratories, Inc.) and a chemical-lysis based QIAamp DNA mini kit (Qiagen, Inc.), following the manufacturer's protocol. Upon finishing, the purified DNA was rinsed in $100 \mu$ l elution buffer ( $\mathrm{pH}$ 8.0) and its concentration was measured using Qubit 2.0 (Thermo Fisher Scientific, Inc.). The microbiome DNA was PCR-amplified using barcoded primers spanning the V4 variable region of the 16s rRNA gene, as previously described (19). Barcoded PCR products from all samples were pooled at approximately equal molar DNA concentrations and sequenced on an Illumina MiSeq (Illumina Inc.) by the Albert Einstein Epigenomics Shared Facility using paired-end reads.

The short Illumina reads were processed for an operational taxonomic unit (OTU) classification according to previously described methods (20-23). Compositions of microbiome communities were summarized by a proportion at the genus level.

Statistical analysis. The OTU results of all the samples in one group are presented in Fig. 1 and were based on individual OTUs. The relative levels of the species were plotted with GraphPad software (version 6; GraphPad Software, Inc.). Heatmaps were generated from the specimen and samples. Unpaired Student's t-tests were used to identify significant differences between groups. $\mathrm{P}<0.05$ was considered to indicate a statistically significant difference. $\chi^{2}$ tests were used to compare the main intestinal flora types between the KSD and HLT groups.

To determine the major intestinal flora types in the experiment and control groups, age, sex, presence of diabetes mellitus (DM) and body mass index (BMI) were added to the independent logistic regression model of KSD associated with Bacteroides, Pseudomonas and Blautia. Unpaired Student's t-tests were also used to compare the mean abundance levels of Bacteroides, Pseudomonas and Blautia with DM, hypertension and BMI. A non-parametric test was used to compare the COKS, UAKS and CCKS groups. An unpaired two-tailed Student t-test was used to compare the difference between two groups. One-way ANOVA followed by Tukey's post hoc test was used for multiple comparisons. The statistical methods used in Table III were ANOVAs (one-way) and post hoc Tukey's tests. All examinations were bilateral $(\mathrm{P}<0.05)$. Data were statistically analyzed using SPSS v.20 (IBM Corp.).

\section{Results}

KSD and intestinal dysbacteriosis. Fecal samples were collected from 91 patients with KSD and 75 HLT subjects (patient information is presented in Table I). A total of 178 genera were identified by 16 s rRNA sequencing, and the top 10 bacteria with the highest levels were statistically analyzed. At the phylum level, the abundance of Firmicutes and Bacteroides was highest in the KSD group. Moreover, the HLT group exhibited higher abundance levels of Actinobacteria (Fig. 1A and B). At the genus level, the abundance of Bacteroides and Prevotella-9 was highest in the KSD group, accounting for $31 \%$ of all bacteria present. The abundance of Bacteroides in the KSD group was higher compared with that in the HLT group (22.2 vs. $3.6 \%$; $\mathrm{P}<0.001)$. The Prevotella-9 abundance was 4.65 -fold higher in the KSD group compared 


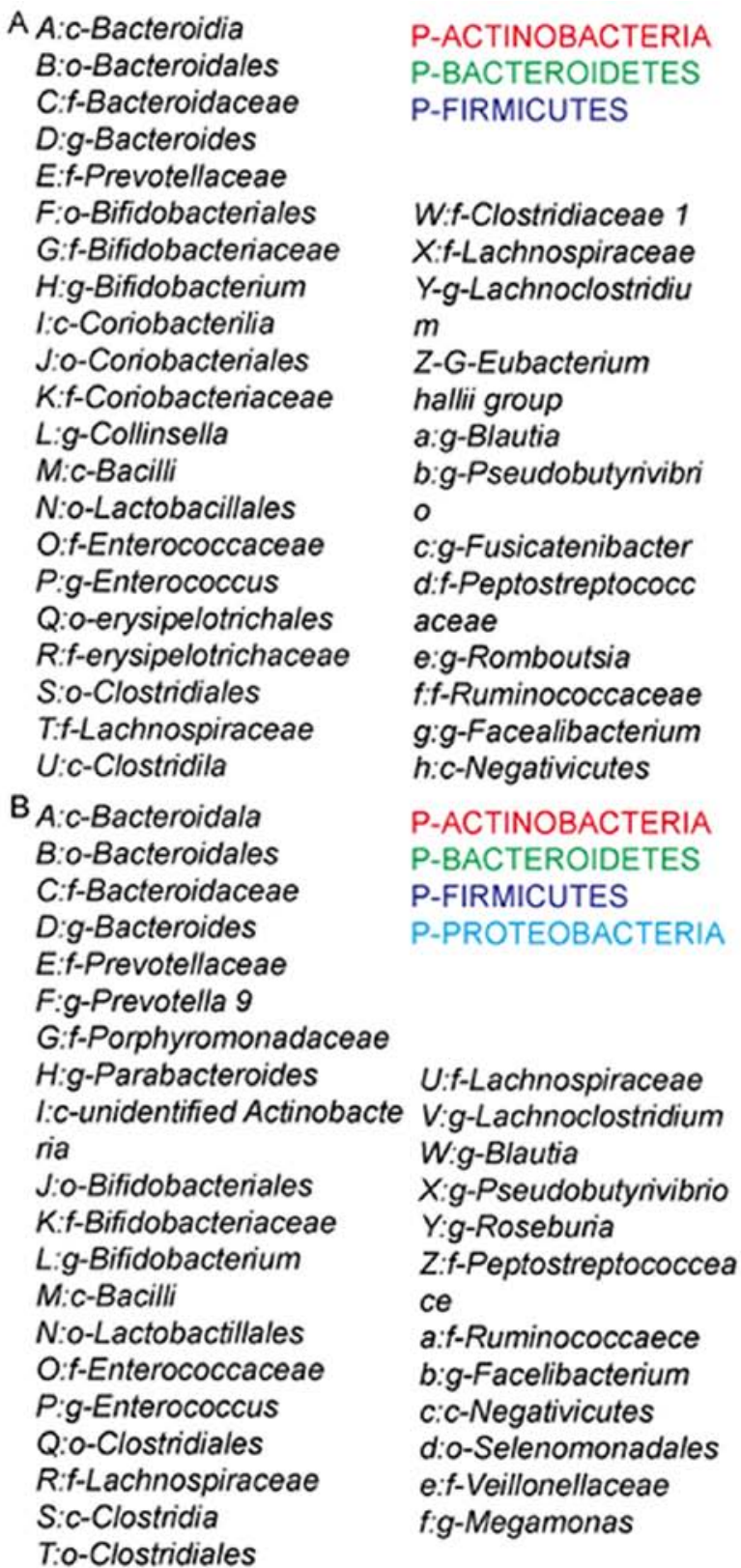

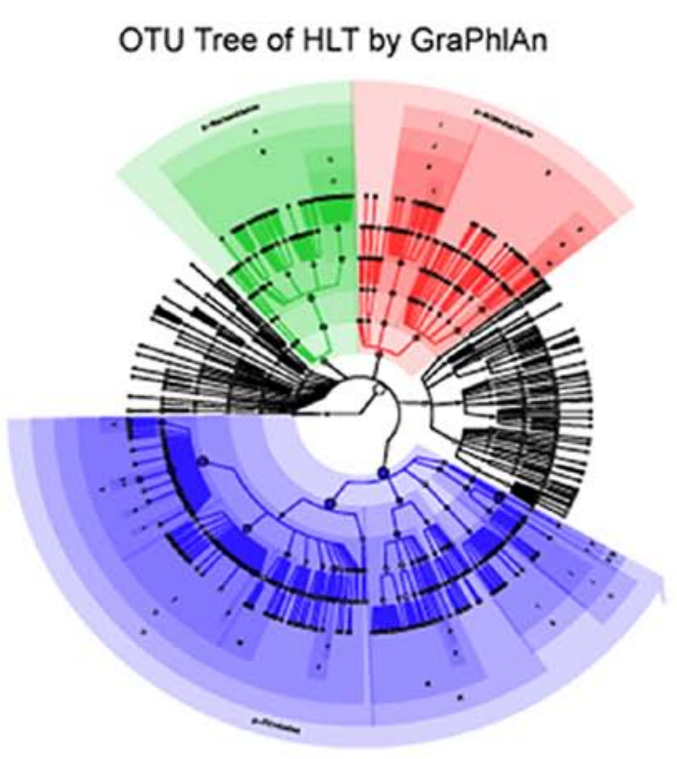

OTU Tree of KSD by GraPhIAn

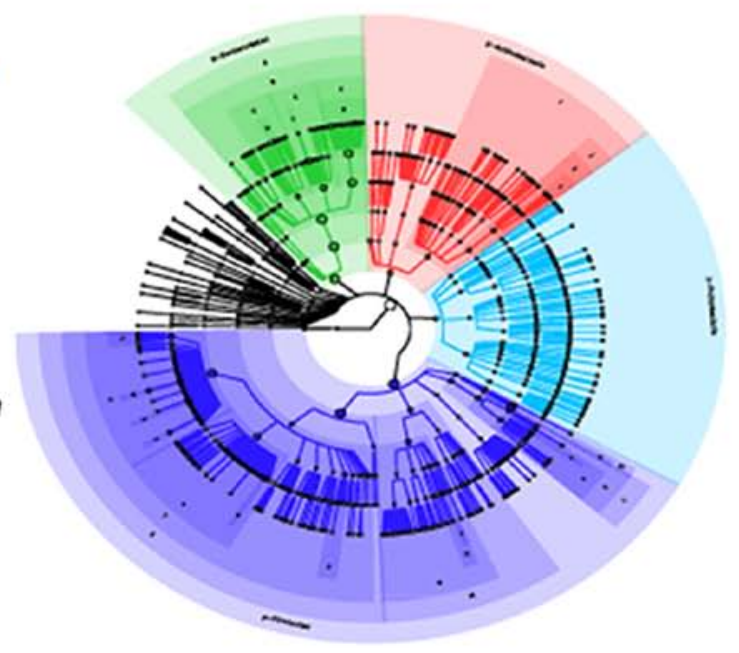

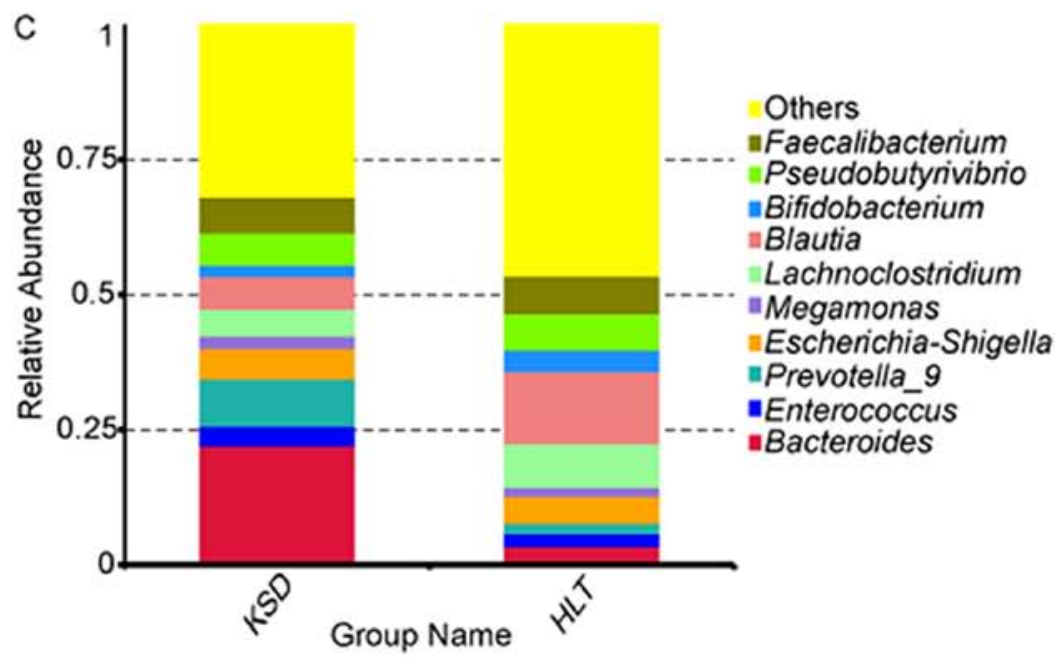

Figure 1. Differences in the abundance of intestinal flora between the KSD and HLT groups. (A) OTU results of 75 samples from the KSD group are presented, using GraPhlAn and the OTU table. (B) OTU results of 91 samples from the HLT group are presented using GraPhlAn and the OTU table. (C) Top ten differences in intestinal flora between the KSD and HLT groups at the genus level. KSD, kidney stone disease; HKT, healthy control; OTU, operational taxonomic unit. 
Table I. Key characteristics of the KSD and HLT groups.

\begin{tabular}{lccc}
\hline Variable & KSD & HLT & P-value \\
\hline Age, years & $56.3 \pm 11.4$ & $57.0 \pm 13.3$ & 0.695 \\
Sex & & & 0.445 \\
Female & 43 & 31 & \\
Male & 48 & 44 & \\
BMI & $24.7 \pm 3.4$ & $25.1 \pm 4.1$ & 0.419 \\
Comorbidities & & & \\
DM & $11 \pm 12$ & $10 \pm 13$ & 0.810 \\
HTN & $23 \pm 25$ & $23 \pm 30$ & 0.210 \\
\hline
\end{tabular}

Data are presented as the $\mathrm{n}$ or mean $\pm \mathrm{SD}$. Comparisons were made using $\chi^{2}$ and Student's t-test. KSD, kidney stone disease; HLT, healthy control; BMI, body mass index; DM, diabetes mellitus; HTN, hypertension.

Table II. Top ten differences of intestinal flora (abundance, \%) between KSD and HLT groups at the genus level.

\begin{tabular}{lccr}
\hline Genus & Case & Control & P-value \\
\hline Bacteroides & $0.222 \pm 0.183$ & $0.036 \pm 0.057$ & $<\mathbf{0 . 0 0 1}$ \\
Enterococcus & $0.036 \pm 0.104$ & $0.024 \pm 0.099$ & 0.474 \\
Prevotella_9 & $0.088 \pm 0.152$ & $0.019 \pm 0.069$ & $<\mathbf{0 . 0 0 1}$ \\
EscherichiaShigella & $0.057 \pm 0.097$ & $0.049 \pm 0.104$ & 0.623 \\
Megamonas & $0.023 \pm 0.054$ & $0.018 \pm 0.074$ & 0.633 \\
Lachnoclostridium & $0.050 \pm 0.043$ & $0.080 \pm 0.094$ & $\mathbf{0 . 0 1 3}$ \\
Blautia & $0.060 \pm 0.064$ & $0.134 \pm 0.072$ & $<\mathbf{0 . 0 0 1}$ \\
Bifidobacterium & $0.022 \pm 0.050$ & $0.041 \pm 0.072$ & $\mathbf{0 . 0 4 3}$ \\
Pseudobutyrivibrio & $0.060 \pm 0.071$ & $0.066 \pm 0.063$ & 0.530 \\
Faecalibacterium & $0.067 \pm 0.065$ & $0.070 \pm 0.063$ & 0.721 \\
\hline
\end{tabular}

Data are presented as the mean $\pm \mathrm{SD}$. Comparisons were made using Student's t-test. Bold values indicate statistical significance $(\mathrm{P}<0.05)$. KSD, kidney stone disease; HLT, healthy control.

with that in the HLT group ( 8.8 vs. $1.9 \%$; $\mathrm{P}<0.001)$. The two bacteria with the highest levels in the HLT group were Blautia and Lachnoclostridium, accounting for $21 \%$ of total bacteria (Fig. 1C; Table II). The Blautia abundance was 2.12-fold higher in the HLT group compared with the KSD group (13.4 vs. 6.0\%; $\mathrm{P}<0.001$ ). The abundance of Lachnoclostridium was 1.58 -fold higher in the HLT group compared with the KSD group (8.0 vs. $5.0 \%$; $\mathrm{P}=0.013$ Fig. $1 \mathrm{C}$; Table II). High abundance of Prevotella- $9(\mathrm{OR}=3.78 ; \mathrm{P}<0.001)$ and Blontia $(\mathrm{OR}=-3.04, \mathrm{P}=0.002)$, and low abundance of Bacteroides $(\mathrm{OR}=6.86 ; \mathrm{P}<0.001)$ were independent influencing factors in the KSD group according to multivariate analysis (Table II).

Different types of intestinal dysbacteriosis are associated with different types of KSs. Among the COKS, UAKS and CCKS groups, female morbidity was highest in the CCKS group $(\mathrm{n}=5 ; \mathrm{P}=0.034$; Table III). The classification of stones was based on the components with the largest proportion (18). The
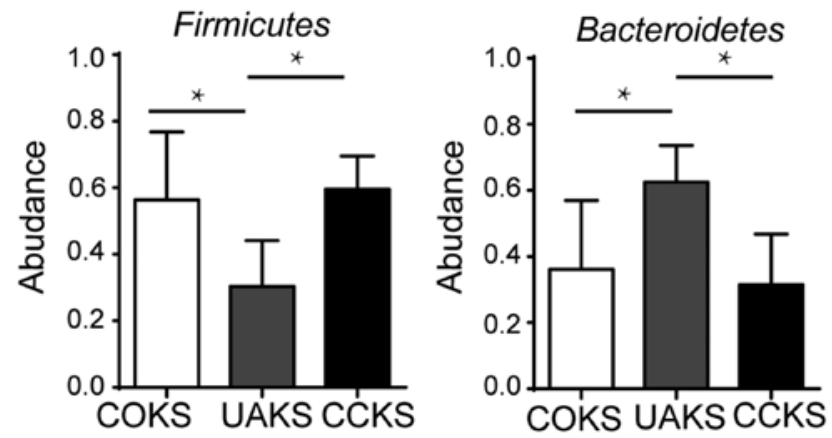

Figure 2. Differences in the abundance of intestinal flora among the COKS UAKS and CCKS groups at the phylum level. After analysis among the three groups, graphs depicting the statistical significance were created. ${ }^{*} \mathrm{P}<0.05$. COKS, calcium oxalate kidney stones; UAKS, uric acid kidney stones; CCKS, carbonate apatite kidney stones.

majority of the enrolled patients had a urinary tract infection when they were hospitalized, but urea-positive bacteria were not found in clinical urine bacterial culture. The abundance of Firmicutes (56.4 vs. 30.4\%; $\mathrm{P}=0.029)$ and Bacteroides (36.2 vs. $62.6 \% ; \mathrm{P}=0.009)$ differed significantly between the COKS and UAKS groups. In the CCKS and UAKS groups, there were significant differences in the abundance levels of Firmicutes (59.5 vs. 30.4\%; $\mathrm{P}=0.014$ ) and Bacteroidetes (31.5 vs. $62.6 \% ; \mathrm{P}=0.006)$. There was no significant difference in the abundance of Firmicutes (56.4 vs. 59.5\%; P>0.05) and Bacteroides ( 36.2 vs. $31.5 \%$; $\mathrm{P}>0.05$ ) between the COKS and CCKS groups (Fig. 2).

Among COKS, UAKS and CCKS groups, Prevotella had the highest abundance in the UAKS group, Pseudobutyrivibrio and Lachnoclostridium had the highest abundance in the COKS group, and Ruminococcus- 2 had the lowest abundance in the UAKS group (Fig. 3). Moreover, no significant differences in the abundance of bacteria were identified between the COKS and CCKS groups containing calcium (all P>0.05; Fig. 4). The Prevotella abundance levels differed between the non-calcium-containing UAKS and the calcium-containing COKS and CCKS groups (32.2 vs. 8.6 and $2.0 \%$, respectively; all $\mathrm{P}<0.005$; Fig. 4). In addition, there were differences in abundance levels between the COKS and the UAKS groups for Pseudobutyrivibrio, Lachnoclostridium, Ruminococcus and Fusicatenibacter (7.7 vs. $2.9 \%, 4.5$ vs. $1.9 \%, 1.4$ vs. $0.2 \%$ and 1.2 vs. $0.6 \%$, respectively; all $\mathrm{P}<0.05$; Fig. 4). There were differences between the three species of Anaerotruncus, Tyzzerell-4 and Turicibacter, but the abundance level was very low (data not shown).

The differences in gastrointestinal flora were analyzed and compared between men and women (Table IV). There were no statistically significant differences in gastrointestinal flora between the KSD and the HLT groups under the phylum level classification (all $\mathrm{P}>0.05$; data not shown). Under the genus classification, significant differences were observed in the KSD groups between men and women. Statistically significant differences were identified among Collinsella, Peptostreptococcus, Sutterella, Barnesiella, Peptococcus, Senegalimassilia, Butyricimonas, Bilophila, Ruminiclostridium-9, Coprobacter, Mogibacterium and Cupriavidus (all $\mathrm{P}<0.05$; Table IV). The abundance levels of 
Table III. Clinical characteristics of the COKS, UAKS and CCKS stone groups.

\begin{tabular}{|c|c|c|c|c|}
\hline Variable & COKS & UAKS & CCKS & P-value \\
\hline Age, years & $56.4 \pm 10.6$ & $46.0 \pm 4.8$ & $55.7 \pm 13.6$ & 0.043 \\
\hline Sex & & & & 0.034 \\
\hline Female & 9 & 6 & 15 & \\
\hline Male & 33 & 6 & 3 & \\
\hline BMI & $24.7 \pm 2.8$ & $28.8 \pm 4.7$ & $24.1 \pm 2.8$ & 0.223 \\
\hline Urine $\mathrm{pH}$ & $5.7 \pm 0.6$ & $6.1 \pm 1.0$ & $6.9 \pm 0.6$ & 0.434 \\
\hline \multicolumn{5}{|c|}{ Biochemical index } \\
\hline $\mathrm{Ca}$ & $2.33 \pm 0.11$ & $2.25 \pm 0.11$ & $2.20 \pm 0.12$ & 0.328 \\
\hline $\mathrm{P}$ & $1.10 \pm 0.16$ & $1.04 \pm 0.15$ & $1.17 \pm 0.12$ & 0.651 \\
\hline $\mathrm{Mg}$ & $0.99 \pm 0.07$ & $1.36 \pm 0.64$ & $1.02 \pm 0.07$ & 0.448 \\
\hline $\mathrm{TC}$ & $7.76 \pm 5.36$ & $5.05 \pm 1.32$ & $4.68 \pm 1.83$ & 0.292 \\
\hline TG & $3.33 \pm 2.04$ & $3.52 \pm 1.34$ & $2.30 \pm 2.42$ & 0.151 \\
\hline HDL & $1.44 \pm 0.37$ & $1.02 \pm 0.24$ & $1.29 \pm 0.53$ & 0.471 \\
\hline LDL & $3.02 \pm 1.17$ & $2.31 \pm 0.24$ & $2.65 \pm 1.08$ & 0.252 \\
\hline CRP & $7.25 \pm 9.21$ & $4.77 \pm 3.97$ & $8.41 \pm 7.81$ & 0.439 \\
\hline
\end{tabular}

Data are presented as the $\mathrm{n}$ or mean $\pm \mathrm{SD}$. Comparisons were made using ANOVAs (one-way) and post hoc Tukey's tests. Bold values indicate statistical significance $(\mathrm{P}<0.05)$. COKS, calcium oxalate kidney stones; UAKS, uric acid kidney stones; CCKS, carbonated apatite kidney stones; TC, serum total cholesterol; TG, triglyceride; HDL, high-density lipoprotein; LDL, low-density lipoprotein; CRP, C-reactive protein.
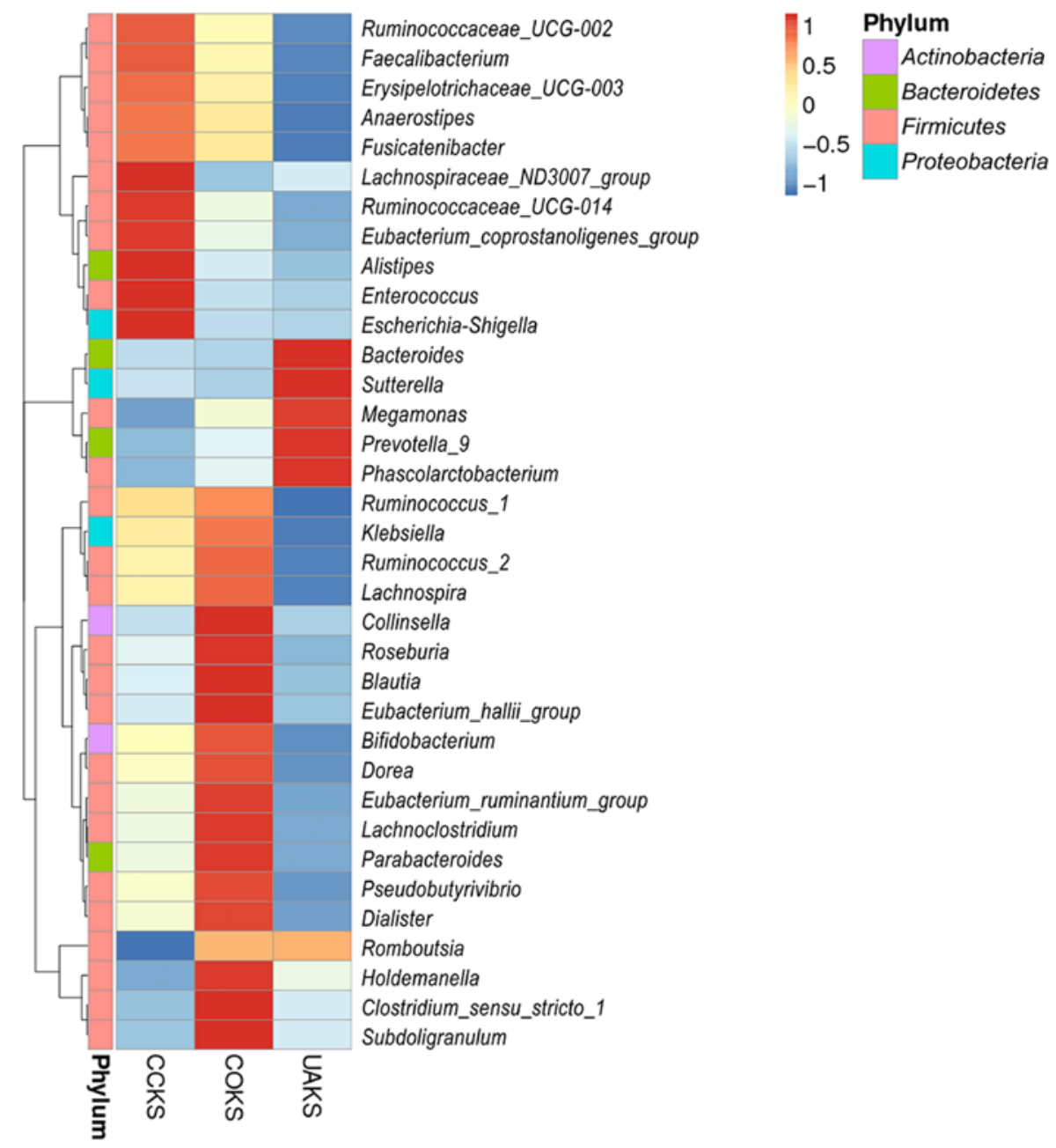

Eubacterium_coprostanoligenes_group

Alistipes

Megamonas

Prevotella_9

Phascolarctobacterium

Ruminococcus_1

Blautia

Eubacterium_hallii group

Bifidobacterium

Lachnoclostridium

Parabacteroides

Pseudobutyrivibrio

Dialister

Clostridium_sensu_stricto_1

紊 ก

Figure 3. A heat map comparing the intestinal flora between the COKS, UAKS and CCKS groups at the genus level. COKS, calcium oxalate kidney stones; UAKS, uric acid kidney stones; CCKS, carbonate apatite kidney stones. 

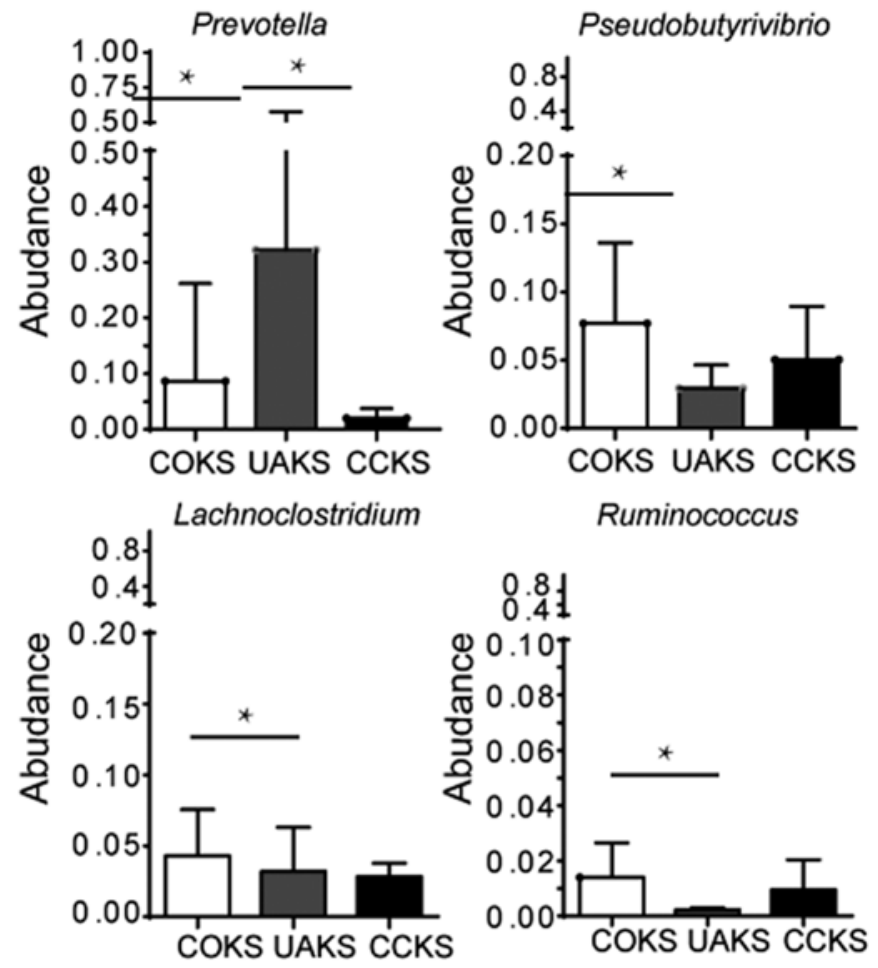

Ruminococcus

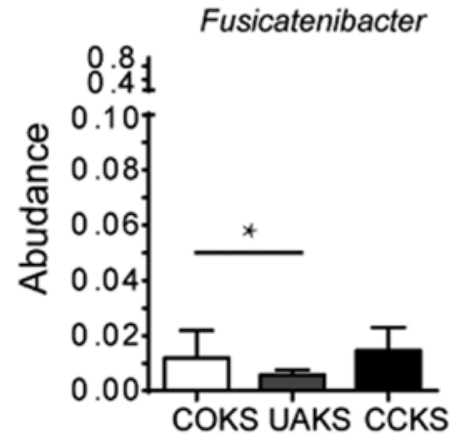

Figure 4. Differences in the abundance of intestinal flora between the COKS, UAKS and CCKS groups at the genus level. After analysis among the three groups, graphs depicting the statistical significance were created. ${ }^{*} \mathrm{P}<0.05$. COKS, calcium oxalate kidney stones; UAKS, uric acid kidney stones; CCKS, carbonate apatite kidney stones.

Collinsella (0.7 vs. $0.2 \% ; \mathrm{P}=0.031$ ), Sutterella (0.3 vs. $0.1 \%$; $\mathrm{P}=0.028$ ) and others (data not shown) were very low. In the HLT group, there were significant differences among unidentified-Lachnospiraceae, Paraprevotella, Haemophilus, Sphingomonas, Ruminiclostridium-1, Streptomyces, Johnsonella and Acholeplasma (all $\mathrm{P}<0.05$; Table IV), but the abundance levels were very low (data not shown).

\section{Discussion}

The composition of KSs is complex and diverse, and pathogenic mechanisms are affected by multiple factors, with diet being the most important factor affecting the formation of KSs (4-6,24,25). The differences in ethnicity and dietary structure markedly affect the composition and function of intestinal ecosystems (26-29). The present study demonstrated that patients with KSs had intestinal dysbacteriosis, and Oxalobacter levels were upregulated in patients with COKS. However, the prevalence of specific bacteria was not obvious among other patients with KSD. The present study analyzed the differences in gastrointestinal bacteria among patients with KSD, and performed further comparative analyses of bacterial abundance and the specific composition of KSs.

The mechanism of KS formation is complex. A variety of stone compositions contribute to metabolic regulation under control of the susceptibility gene (30). Similar to other researchers $(4-6,25)$, the present results suggested that diet was one of the most important factors affecting the formation of kidney stones. Endogenous and exogenous oxalic acid metabolic disorders lead to formation of COKS, with Oxalobacter being one of the most important contributors (17). Few studies have reported other types of bacteria causing gastrointestinal disorders to be involved in KS formation.

It was hypothesized that the abnormal changes in metabolic products and bacterial intestinal colonization may trigger mucosal immune inflammation mechanisms, which may cause further changes in the body's renal function when intestinal dysbacteriosis is present. When the levels of the components of kidney stones increases, renal tubular epithelial injury, inflammatory responses and calculus susceptibility gene regulation occur, eventually leading to KS formation $(31,32)$.

The high incidence and recurrence of KSD may be closely associated with ethnicity and diet; thus, it is important to investigate changes in intestinal dysbacteriosis (33). In the present study, the abundance of Bacteroides and Prevotella in the KSD group was significantly higher compared with that in the HLT group (6.1- and 4.6-fold, respectively). The trend of the obtained results was consistent with that reported by Stern et al (2) (6.1- vs. 3.4-fold and 4.6- vs. 2.8-fold, respectively), and variations may be due to differences in ethnicity and dietary cultures of the patients recruited. In addition, Lachnoclostridium, Blautia and Bifidobacterium were 1.5-, 2.1- and 1.9-fold higher, respectively, in patients compared with the HLT group.

The differences between the CCKS, UAKS and COKS groups were further analyzed. No lower abundance levels of Oxalobacter were found in the KSD group when compared with the HLT group, which was consistent with the findings of Suryavanshi et al (17). However, in the present study, statistically significant differences in the levels of Oxalobacter between these two groups were not observed, but there were significant differences in Prevotella- 9 abundance between the calcium-containing COKS and CCKS groups and the non-calcium-containing UAKS group. Prevotella-9 is positively correlated with serum uric acid levels, while UAKS are a product of metabolic disorders of uric acid (34). Thus, it was suggested that abnormally high abundance of Prevotella-9 may lead to the formation of uric acid stones. However, it should be noted that Stern et al (2) did not identify any significant differences in bacteria when comparing uric acid and non-UAKS, which may be attributed to the daily high-fiber diet of the study population. Additionally, in the present study, differences were observed in the abundance levels of Pseudobutyrivibrio, Lachnoclostridium and Ruminococcus 2 between the COKS and UAKS groups, all of which belong to the Helicobacter pylori family. One of the 
Table IV. Sex differences in KSD groups (male, $n=48$; female, $n=43$ ) and HLT groups (male, $n=44$; female, $n=31$ ) under genus classification.

\begin{tabular}{lccc} 
A, KSD groups & & \\
\hline Taxonomy & Male & Female & P-value \\
\hline Collinsella & $427.631 \pm 946.67$ & $115.771 \pm 226.548$ \\
Peptostreptococcus & $2.501 \pm 5.772$ & $0.491 \pm 0.883$ & $\mathbf{0 . 0 3 1}$ \\
Sutterella & $84.771 \pm 132.766$ & $207.531 \pm 334.135$ & $\mathbf{0 . 0 2 1}$ \\
Barnesiella & $24.150 \pm 59.532$ & $88.811 \pm 169.650$ & $\mathbf{0 . 0 2 8}$ \\
Peptococcus & $1.791 \pm 3.175$ & $0.671 \pm 1.796$ & $\mathbf{0 . 0 2 2}$ \\
Senegalimassilia & $4.171 \pm 9.924$ & $0.951 \pm 2.663$ & $\mathbf{0 . 0 4 0}$ \\
Butyricimonas & $9.001 \pm 14.388$ & $27.441 \pm 55.455$ \\
Bilophila & $11.351 \pm 12.790$ & $28.491 \pm 46.608$ \\
Ruminiclostridium-9 & $15.311 \pm 19.333$ & $33.811 \pm 46.321$ \\
Coprobacter & $2.981 \pm 8.721$ & $12.001 \pm 26.265$ \\
Mogibacterium & $0.331 \pm 0.753$ & $0.051 \pm 0.213$ & $\mathbf{0 . 0 3 5}$ \\
Cupriavidus & $0.081 \pm 0.279$ & $0.001 \pm 0.000$ & $\mathbf{0 . 0 4 0}$ \\
\hline
\end{tabular}

B, HLT groups

\begin{tabular}{lccc}
\hline Taxonomy & Male & Female & P-value \\
\hline unidentified-Lachnospiraceae & $19.141 \pm 29.890$ & $49.190 \pm 79.644$ & $6.441 \pm 6.730$ \\
Paraprevotella & $23.720 \pm 44.767$ & $4.130 \pm 5.780$ & $\mathbf{0 . 0 4 9}$ \\
Haemophilus & $28.741 \pm 72.885$ & $0.031 \pm 0.177$ & $\mathbf{0 . 0 1 6}$ \\
Sphingomonas & $0.261 \pm 0.658$ & $0.001 \pm 0.000$ & $\mathbf{0 . 0 3 3}$ \\
Ruminiclostridium-1 & $0.140 \pm 0.351$ & $0.001 \pm 0.000$ & $\mathbf{0 . 0 3 8}$ \\
Streptomyces & $0.231 \pm 0.611$ & $0.001 \pm 0.000$ & $\mathbf{0 . 0 1 3}$ \\
Johnsonella & $0.161 \pm 0.433$ & $0.001 \pm 0.000$ & $\mathbf{0 . 0 1 7}$ \\
Acholeplasma & $0.121 \pm 0.324$ & $\mathbf{0 . 0 1 8}$ \\
\hline
\end{tabular}

The minimum measured abundance value was 1 . Comparisons were made using $\chi^{2}$ test and Student's t-test. Bold values indicate statistical significance $(\mathrm{P}<0.05)$. KSD, kidney stone disease; HTL, healthy control.

major metabolites is short-chain fatty acids, which participates in the metabolism (35) and inflammatory responses of the body $(36,37)$. It was hypothesized that the bacteria present in intestinal dysbacteriosis may interfere with the body's normal inflammatory response and material metabolism via uric acid short-chain fatty acid metabolic disorders, which ultimately lead to calcium and UAKS formation in the kidney.

In general, the composition of intestinal microbiota changes according to age, diet and drug use, and differs across ethnicities and geographic regions $(2,3,5,8,17)$. Loss of Oxalobacter formigeneses is hypothesized to be a factor that produces COKS, as Oxalobacter formigeneses metabolizes oxalate in the intestine; however, several studies, including the present, question the validity of this hypothesis $(2,38)$. The mechanism underlying the formation of calculi has yet to be fully elucidated. Further extensive basic research and clinical data are required for confirmation. Although individuals residing in the same area tend to consume similar foods, individual dietary variations also affect the statistical results $(4,6)$. The present study randomly recruited participants in both the KSD and HLT groups. The stones collected were mostly of mixed composition. The main stone composition was based on infrared spectrum analysis. The participants did not strictly control their daily diets in the present study. Since some patients opted for holmium laser lithotripsy and some did not agree to undergo stone composition analysis, and thus, there was an insufficient number of patients in the COKS, UAKS and CCKS groups to establish statistical significance.

In conclusion, the present study indicated a key association between specific KS components and intestinal flora, providing a theoretical basis for novel treatment strategies for KSs. Moreover, the results of the present study indicated that differences and interactions between bacteria might predict specific types of urolithiasis.

\section{Acknowledgements}

Not applicable. 


\section{Funding}

The present study was supported by the National Natural Science Foundation of Heilongjiang Province (grant no. H201407).

\section{Availability of data and materials}

All data generated or analyzed during this study are included in this published article.

\section{Authors' contributions}

XL was responsible for designing the experiments, and analyzing and interpreting the data. EZ was responsible for compiling articles, performing the experiments and analyzing the data. WZ, BG, BY and WW collected clinical specimens and interpreted the data. All authors read and approved the final manuscript.

\section{Ethics approval and consent to participate}

The protocol of the present study was approved by the Ethics Committee of the Second Affiliated Hospital of Harbin Medical University (approval no. 2015-yan-221) and complied with the World Medical Association Declaration of Helsinki regarding ethical conduct of research involving human subjects. The study was performed in accordance with the approved local guidelines and regulations, and written informed consent was obtained from all the participants.

\section{Patient consent for publication}

Not applicable.

\section{Competing interests}

The authors declare that they have no competing interests.

\section{References}

1. Scales CD Jr, Smith AC, Hanley JM and Saigal CS; Urologic Diseases in America Project: Prevalence of kidney stones in the United States. Eur Urol 62: 160-165, 2012.

2. Stern JM, Moazami S, Qiu Y, Kurland I, Chen Z, Agalliu I, Burk R and Davies KP: Evidence for a distinct gut microbiome in kidney stone formers compared to non-stone formers. Urolithiasis 44 399-407, 2016.

3. Daudon M, Dore JC, Jungers P and Lacour B: Changes in stone composition according to age and gender of patients: A multivariate epidemiological approach. Urol Res 32: 241-247, 2004.

4. Taylor EN, Stampfer MJ and Curhan GC: Dietary factors and the risk of incident kidney stones in men: New insights after 14 years of follow-up. J Am Soc Nephrol 15: 3225-3232, 2004.

5. Tracy CR, Best S, Bagrodia A, Poindexter JR, Adams-Huet B, Sakhaee K, Maalouf N, Pak CY and Pearle MS: Animal protein and the risk of kidney stones: A comparative metabolic study of animal protein sources. J Urol 192: 137-141, 2014.

6. Borghi L, Schianchi T, Meschi T, Guerra A, Allegri F, Maggiore U and Novarini A: Comparison of two diets for the prevention of recurrent stones in idiopathic hypercalciuria. N Engl J Med 346: 77-84, 2002.

7. Brunkwall L and Orho-Melander M: The gut microbiome as a target for prevention and treatment of hyperglycaemia in type 2 diabetes: From current human evidence to future possibilities. Diabetologia 60: 943-951, 2017.
8. Karlsson FH, Tremaroli V, Nookaew I, Bergstrom G, Behre CJ Fagerberg B, Nielsen J and Backhed F: Gut metagenome in European women with normal, impaired and diabetic glucose control. Nature 498: 99-103, 2013.

9. Qin J, Li Y, Cai Z, Li S, Zhu J, Zhang F, Liang S, Zhang W, Guan Y, Shen D, et al: A metagenome-wide association study of gut microbiota in type 2 diabetes. Nature 490: 55-60, 2012.

10. Dumas ME, Barton RH, Toye A, Cloarec O, Blancher C, Rothwell A, Fearnside J, Tatoud R, Blanc V, Lindon JC, et al: Metabolic profiling reveals a contribution of gut microbiota to fatty liver phenotype in insulin-resistant mice. Proc Natl Acad Sci USA 103: 12511-12516, 2006.

11. Pedersen HK, Gudmundsdottir V, Nielsen HB, Hyotylainen T, Nielsen T, Jensen BA, Forslund K, Hildebrand F, Prifti E, Falony G, et al: Human gut microbes impact host serum metabolome and insulin sensitivity. Nature 535: 376-381, 2016.

12. Le Chatelier E, Nielsen T, Qin J, Prifti E, Hildebrand F, Falony G, Almeida M, Arumugam M, Batto JM, Kennedy S, et al: Richness of human gut microbiome correlates with metabolic markers. Nature 500: 541-546, 2013.

13. Ussar S, Griffin NW, Bezy O, Fujisaka S, Vienberg S, Softic S, Deng L, Bry L, Gordon JI and Kahn CR: Interactions between gut microbiota, host genetics and diet modulate the predisposition to obesity and metabolic syndrome. Cell Metab 22: 516-530, 2015.

14. Tang WH, Wang Z, Shrestha K, Borowski AG, Wu Y, Troughton RW, Klein AL and Hazen SL: Intestinal microbiota-dependent phosphatidylcholine metabolites, diastolic dysfunction, and adverse clinical outcomes in chronic systolic heart failure. J Card Fail 21: 91-96, 2015.

15. Wang Z, Klipfell E, Bennett BJ, Koeth R, Levison BS, Dugar B, Feldstein AE, Britt EB, Fu X, Chung YM, et al: Gut flora metabolism of phosphatidylcholine promotes cardiovascular disease. Nature 472: 57-63, 2011.

16. Zhu W, Gregory JC, Org E, Buffa JA, Gupta N, Wang Z, Li L, Fu X, Wu Y, Mehrabian M, et al: Gut microbial metabolite TMAO enhances platelet hyperreactivity and thrombosis risk. Cell 165: 111-124, 2016.

17. Suryavanshi MV, Bhute SS, Jadhav SD, Bhatia MS, Gune RP and Shouche YS: Hyperoxaluria leads to dysbiosis and drives selective enrichment of oxalate metabolizing bacterial species in recurrent kidney stone endures. Sci Rep 6: 34712, 2016.

18. Mandel NS, Mandel IC and Kolbach-Mandel AM: Accurate stone analysis: The impact on disease diagnosis and treatment. Urolithiasis 45: 3-9, 2017.

19. Wang Y and Qian PY: Conservative fragments in bacterial $16 \mathrm{~S}$ rRNA genes and primer design for 16S ribosomal DNA amplicons in metagenomic studies. PLoS One 4: e7401, 2009.

20. Smith BC, McAndrew T, Chen Z, Harari A, Barris DM, Viswanathan S, Rodriguez AC, Castle P, Herrero R, Schiffman M and Burk RD: The cervical microbiome over 7 years and a comparison of methodologies for its characterization. PLoS One 7: e40425, 2012.

21. Caporaso JG, Kuczynski J, Stombaugh J, Bittinger K, Bushman FD, Costello EK, Fierer N, Peña AG, Goodrich JK, Gordon JI, et al: QIIME allows analysis of high-throughput community sequencing data. Nat Methods 7: 335-336, 2010.

22. Edgar RC: Search and clustering orders of magnitude faster than BLAST. Bioinformatics 1: 2460-2461, 2010.

23. Matsen FA, Kodner RB and Armbrust EV: Pplacer: Linear time maximum-likelihood and Bayesian phylogenetic placement of sequences onto a fixed reference tree. BMC Bioinform 11: 538, 2010.

24. Org E, Blum Y, Kasela S, Mehrabian M, Kuusisto J, Kangas AJ, Soininen P, Wang Z, Ala-Korpela M, Hazen SL, et al: Relationships between gut microbiota, plasma metabolites, and metabolic syndrome traits in the METSIM cohort. Genome Biol 18: 70, 2017.

25. Pak CY: Kidney stones. Lancet 351: 1797-1801, 1998.

26. Parks BW, Nam E, Org E, Kostem E, Norheim F, Hui ST, Pan C, Civelek M, Rau CD, Bennett BJ, et al: Genetic control of obesity and gut microbiota composition in response to high-fat, high-sucrose diet in mice. Cell Metab 17: 141-152, 2013.

27. Wu GD, Chen J,Hoffmann C, Bittinger K, Chen YY, Keilbaugh SA, Bewtra M, Knights D, Walters WA, Knight R, et al: Linking long-term dietary patterns with gut microbial enterotypes. Science 334: 105-108, 2011.

28. Daniel H, Gholami AM, Berry D, Desmarchelier C, Hahne H, Loh G, Mondot S, Lepage P, Rothballer M, Walker A, et al: High-fat diet alters gut microbiota physiology in mice. ISME J 8: 295-308, 2014. 
29. David LA, Maurice CF, Carmody RN, Gootenberg DB, Button JE Wolfe BE, Ling AV, Devlin AS, Varma Y, Fischbach MA, et al: Diet rapidly and reproducibly alters the human gut microbiome. Nature 505: 559-563, 2014.

30. Palsson R, Indridason OS, Edvardsson VO and Oddsson A: Genetics of common complex kidney stone disease: Insights from genome-wide association studies. Urolithiasis 47: 11-21, 2019.

31. Ratajczak W, Rył A, Mizerski A, Walczakiewicz K, Sipak O and Laszczyńska M: Immunomodulatory potential of gut microbiome-derived short-chain fatty acids (SCFAs). Acta Biochim Pol 66: 1-12, 2019

32. Okada A, Yasui T, Fujii Y, Niimi K, Hamamoto S, Hirose M, Kojima Y, Itoh Y, Tozawa K, Hayashi Y and Kohri K: Renal macrophage migration and crystal phagocytosis via inflammatory-related gene expression during kidney stone formation and elimination in mice: Detection by association analysis of stone-related gene expression and microstructural observation. J Bone Miner Res 12: 2701-2711, 2010.

33. Fakhoury MQ, Gordon B, Shorter B, Renson A, Borofsky MS, Cohn MR, Cabezon E, Wysock JS and Bjurlin MA: Perceptions of dietary factors promoting and preventing nephrolithiasis: A cross-sectional survey. World J Urol 37: 1723-1731, 2019.
34. Lim MY, Rho M, Song YM, Lee K, Sung J and Ko G: Stability of gut enterotypes in Korean monozygotic twins and their association with biomarkers and diet. Sci Rep 4: 7348, 2014.

35. Teixeira TF, Grzeskowiak L, Franceschini SC, Bressan J, Ferreira CL and Peluzio MC: Higher level of faecal SCFA in women correlates with metabolic syndrome risk factors. Br J Nutr 109: 914-919, 2013.

36. Winter SE and Baumler AJ: Why related bacterial species bloom simultaneously in the gut: Principles underlying the 'like will to like' concept. Cell Microbiol 16: 179-184, 2014.

37. Winter SE and Baumler AJ: Dysbiosis in the inflamed intestine: Chance favors the prepared microbe. Gut Microbes 5: 71-73, 2014.

38. Lee JA and Stern JM: Understanding the link between gut microbiome and urinary stone disease. Curr Urol Rep 20: 19, 2019.

(c) (i) () This work is licensed under a Creative Commons Attribution-NonCommercial-NoDerivatives 4.0 International (CC BY-NC-ND 4.0) License. 